\title{
Increased expression of stathmin and elongation factor $1 a$ in precancerous nodules with telomere dysfunction in hepatitis B viral cirrhotic patients
}

Ei Yong Ahn ${ }^{1,2+}$, Jeong Eun Yoo ${ }^{1,2+}$, Hyungjin Rhee ${ }^{1,2}$, Myung Soo Kim ${ }^{4}$, Junjeong Choi ${ }^{1,3}$, Jung Eun Ko ${ }^{1,2}$, Jee San Lee ${ }^{1,2}$ and Young Nyun Park ${ }^{1,2,5,6^{*}}$

\begin{abstract}
Background: Telomere dysfunction is important in carcinogenesis, and recently, stathmin and elongation factor $1 a$ (EF1a) were reported to be up-regulated in telomere dysfunctional mice.

Methods: In the present study, the expression levels of stathmin and EF1a in relation to telomere length, telomere dysfunction-induced foci (TIF), Y-H2AX, and p21 $1^{\text {WAF1/CIP1 } 1}$ expression were assessed in specimens of hepatitis B virus (HBV)-related multistep hepatocarcinogenesis, including 13 liver cirrhosis specimens, 14 low-grade dysplastic nodules (DN), 17 high-grade DNs, and 14 hepatocellular carcinomas (HCC). Five normal liver specimens were used as controls. TIF were analyzed by telomere fluorescent in situ hybridization (FISH) combined with immunostaining, while the protein

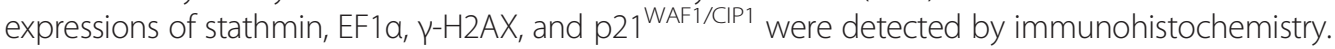

Result: The expressions of stathmin and EF1a gradually increased as multistep hepatocarcinogenesis progressed, showing the highest levels in HCC. Stathmin mRNA levels were higher in high-grade DNs than normal liver and liver cirrhosis, whereas EF1 a mRNA expression did not show such a difference. The protein expressions of stathmin and EFla were found in DNs of precancerous lesions, whereas they were absent or present at very low levels in normal liver and liver cirrhosis. Stathmin histoscores were higher in high-grade DNs and low-grade DNs than in normal liver (all, $P<0.05$ ). EF1a histoscores were higher in high-grade DNs than in normal liver and liver cirrhosis (all, $P<0.05$ ). Stathmin mRNA levels and histoscores, as well as EF1a histoscores (but not mRNA levels), were positively correlated with telomere shortening and $\mathrm{Y}-\mathrm{H} 2 \mathrm{AX}$ labeling index (all, $P<0.05)$. EF1a histoscores were also positively correlated with $\mathrm{TIF}(P<0.001)$. Significantly greater inactivation of p21 $1^{\text {WAF1/CIP1 } 1}$ was observed in low-grade DNs, high-grade DNs, and HCC, compared to

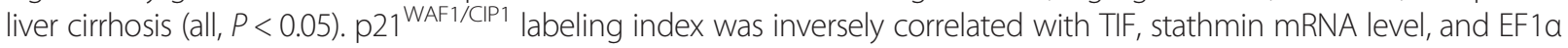
histoscore (all, $P<0.05$ ).
\end{abstract}

Conclusion: Stathmin and EF1a are suggested to be closely related to telomere dysfunction, DNA damage, and inactivation of $\mathrm{p} 21^{\mathrm{WAF} 1 / \mathrm{CIP} 1}$ in HBV-related multistep hepatocarcinogenesis. Accordingly, assessment of stathmin and EF1a levels as a reflection of telomere dysfunction may be helpful in evaluating the biological characteristics of precancerous hepatic nodules in hepatitis B viral cirrhotic patients.

Keywords: Stathmin, Elongation factor 1a, Telomere dysfunction, Hepatocarcinogenesis, Dysplastic nodule, Hepatocellular carcinoma, Hepatitis B virus

\footnotetext{
* Correspondence: young0608@yuhs.ac

${ }^{\dagger}$ Equal contributors

'Department of Pathology, Yonsei University College of Medicine, 250

Seongsan-ro, Seodaemun-gu, Seoul, South Korea

${ }^{2}$ Brain Korea 21 PLUS Project for Medical Science, Seoul, South Korea

Full list of author information is available at the end of the article
} 


\section{Background}

Hepatocellular carcinoma (HCC) is reported as the fifth most common cancer worldwide and the third most common cause of cancer-related deaths [1]. Hepatocarcinogenesis is a multistep process, and has been characterized as the progressive accumulation of genetic and molecular changes in chronic liver disease, which leads to the production of monoclonal populations of dysplastic or transformed hepatocytes. Mutations that impair the DNA damage response pathways facilitate the survival of dysplastic cells with shortened and dysfunctional telomeres, leading to the proliferation of cells with dicentric chromosomes and the accumulation of genomic instability due to breakage-fusion-bridge cycles. In fact, chromosomal instability is characteristic of hepatitis B virus (HBV)-related hepatocarcinogenesis [2]. Liver cirrhosis, a soil for HCC, is characterized by an increase in dysplastic hepatocytes that form dysplastic foci or dysplastic nodules (DNs). Nevertheless, while DNs are considered precancerous lesions of HCC [3], various natural outcomes of DNs have been reported $[4,5]$.

Previously, expression of stathmin and elongation factor $1 \alpha(E F 1 \alpha)$ was reported to be up-regulated in telomere dysfunctional mice [6]. Stathmin, encoded by the human STMN1 gene, is a major microtubule depolymerizing protein involved in cell cycle progression [7]. Stathmin directly interacts with soluble tubulin to form a complex that sequesters free tubulin and hinders the polymerization of microtubules [8]. In mitosis, stathmin is inactivated by phosphorylation to induce microtubule polymerization and the assembly of mitotic spindles; phosphorylated stathmin is reactivated by dephosphorylation, allowing cells to exit mitosis and enter a new interphase [9]. Stathmin, also known as oncoprotein 18, has been reported in several studies to be highly expressed in a wide variety of human cancers, including leukemia, breast, prostate, gastric, and liver cancer, suggesting that stathmin is a key molecule in tumorigenesis [10-13]. Moreover, stathmin overexpression in HCC reportedly increased the malignant potential thereof by regulating cell motility, cell migration, and cell proliferation [12]. Stathmin overexpression in $\mathrm{HCC}$ was also shown to be correlated with poor prognosis $[14,15]$. EF1 $\alpha$ is a translational cofactor of eukaryotic protein synthesis that is important in the elongation of polypeptides. EF1 $\alpha$ carries aminoacyl-tRNA to ribosomes and dissociates after correct codon-anticodon recognition by GTP hydrolysis [16]. Overexpression of EF1 $\alpha$ in human and rodent cells was reported to be associated with increased cell proliferation, oncogenic transformation, and delayed cell senescence [17]. A previous functional study showed that EF1 $\alpha$ interacts with p-Akt to control the activity of p-Akt and regulates the proliferation, survival, and motility of breast cancer cells [18]. Additionally, increased expression of $\mathrm{EF} 1 \alpha$ was reported to be associated with the growth rate of an HCC cell line, but not with apoptosis [19].

The assessment of telomere dysfunction may be helpful in evaluating the biological characteristics of hepatic nodules in cirrhotic patients. However, systemic assessment of the expression levels of stathmin and EF1 $\alpha$ in relation to telomere dysfunction, particularly telomere length and telomerase dysfunction induced foci (TIF), has not been conducted in defined lesions of human multistep hepatocarcinogenesis, including DNs. Accordingly, the present study attempted to evaluate and compare the expression levels of stathmin and EF1 $\alpha$ in relation to telomere length, TIF, $\gamma-\mathrm{H} 2 \mathrm{AX}$, and $\mathrm{p} 21^{\mathrm{WAF} 1 / \mathrm{CIP} 1}$ expression in human HBV-associated multistep hepatocarcinogenesis.

\section{Materials and methods}

\section{Tissue samples and pathological examination}

A total of 58 liver specimens from 24 patients were investigated, including 13 cases of liver cirrhosis, 14 cases of lowgrade DNs, 17 cases of high-grade DNs, and 14 cases of HCC. The specimens were obtained from 19 men and five women whose ages ranged from 32 to 63 years $(51 \pm$ 7.8 years, mean $\pm \mathrm{SD}$ ). They were all serum $\mathrm{HBsAg}$ positive and anti-HCV-negative. The clinicopathological features of the patients are summarized in Table 1. Large nodules, measuring at least $0.5 \mathrm{~cm}$ at their largest dimension, that were apparently distinct from the surrounding cirrhotic parenchyma, in terms of their color, texture, and degree of bulging at the cut surface; HCCs; and corresponding non-neoplastic liver tissue were collected. Half of each nodule was fixed in $10 \%$ buffered formalin, routinely processed, and embedded in paraffin for histological examination. The remaining half of each nodule was snapfrozen in liquid nitrogen and stored at $-80^{\circ} \mathrm{C}$ until ready for use. Hepatocellular nodules were evaluated according to the criteria proposed by the International Consensus Group for Hepatocellular Neoplasia [3]. Differentiation of HCC was determined on the basis of Edmonson and Steiner classification [20]. For comparison, non-neoplastic liver tissues were obtained from 5 patients with metastatic carcinoma. Control subjects were negative for hepatitis virus and showed relatively normal liver histology. The ages of the controls ranged from 42 to 74 years (61 \pm 13.9 years). Fresh frozen specimens were obtained from the Liver Cancer Specimen Bank (part of the National Research Bank Program, Korea Science and Engineering Foundation, Ministry of Science and Technology). This study was approved by the Institutional Review Board of Severance Hospital, Yonsei University College of Medicine, and the requirement for informed consent was waived.

Total RNA extraction and real-time quantitative RT-PCR Total RNA was isolated from human fresh frozen liver tissue samples using TRIzol reagent (Invitrogen, Carlsbad, 
Table 1 Clinical and pathological findings of the patients

\begin{tabular}{|c|c|c|c|c|}
\hline Case no & Age & Sex & Etiology & Pathological diagnosis $^{a}$ \\
\hline 1 & 44 & $\mathrm{~F}$ & HBV & LC, LGDN, HGDN, HGDN, HCC (2) \\
\hline 2 & 52 & M & HBV & LC, LGDN, HGDN, HGDN, HGDN, HGDN, HCC (2), HCC (2) \\
\hline 3 & 60 & $\mathrm{~F}$ & HBV & LC, HGDN, HGDN, HCC (1) \\
\hline 4 & 48 & M & HBV & LC, HGDN, HGDN, HCC (1) \\
\hline 5 & 49 & M & HBV & LC, LGDN, LGDN, LGDN, LGDN, LGDN, LGDN, HCC (3) \\
\hline 6 & 53 & $\mathrm{~F}$ & HBV & LGDN, HGDN \\
\hline 7 & 53 & M & HBV & LGDN, HGDN \\
\hline 8 & 45 & M & HBV & LC, HGDN \\
\hline 9 & 60 & M & HBV & LC, HGDN \\
\hline 10 & 59 & M & HBV & LC, HGDN \\
\hline 11 & 32 & $\mathrm{~F}$ & HBV & LC, LGDN \\
\hline 12 & 48 & M & HBV & LC, LGDN, LGDN \\
\hline 13 & 59 & M & HBV & LC, HCC (1), \\
\hline 14 & 56 & M & HBV & HCC (3) \\
\hline 15 & 41 & M & HBV & HCC (3) \\
\hline 16 & 43 & M & HBV & HCC (3) \\
\hline 17 & 40 & M & HBV & HCC (2) \\
\hline 18 & 60 & M & $\mathrm{HBV}$ & HCC (2) \\
\hline 19 & 54 & $\mathrm{~F}$ & HBV & HCC (2) \\
\hline 20 & 49 & M & HBV & HCC (2) \\
\hline 21 & 51 & M & HBV & LGDN \\
\hline 22 & 63 & M & HBV & HGDN, HGDN \\
\hline 23 & 48 & M & HBV & LC \\
\hline 24 & 48 & M & HBV & LC \\
\hline
\end{tabular}

M, male; F, female; HBV, hepatitis B virus; LGDN, low-grade dysplastic nodule; HGDN, high-grade dysplastic nodule; HCC, hepatocellular carcinoma. ${ }^{a} \mathrm{HCC}$ grading according to the Edmondson and Steiner classification is in parentheses.

CA, USA). The transcription thereof into cDNA was performed using a High Capacity RNA-to-cDNA kit (Applied Biosystems, Foster City, CA, USA). The following TaqMan Gene Expression Assays were purchased from Applied Biosystems: EF1 $\alpha$ (Hs00265885_g1), stathmin (Hs01027 516_g1), and GAPDH (Hs99999905_m1). All measurements were performed in triplicate. The relative expression levels of target mRNAs were normalized to GAPDH mRNA levels.

PCR was performed in triplicate for each cDNA sample using the ABI PRISM 7300 Sequence Detection System (Applied Biosystems). Dual-labeled FAM probes containing a $5{ }^{\prime}$-fluorescent reporter and a $3^{\prime}$ - quencher were used to conduct the PCR experiments.

Immunohistochemical analysis of stathmin, EF1a, p2 $1^{\text {WAF } 1 / C I P 1}$, and $\gamma-\mathrm{H} 2 \mathrm{AX}$

Immunohistochemical staining was performed to detect the expression levels of stathmin, EF $1 \alpha, \mathrm{p} 21^{\mathrm{WAF} 1 / \mathrm{CIP} 1}$, and $\gamma-\mathrm{H} 2 \mathrm{AX}$, as previously described [2]. Details on the antibodies used and antigen-retrieval conditions are summarized in Additional file 1: Table S1.

The staining intensities of stathmin and EF1 $\alpha$ were graded on a scale of $0-3(0$, negative; 1 , weakly positive; 2 , moderately positive; and 3 , strongly positive), and the extent of distribution was rated on a scale of $0-4(0$, positive in $<5 \%$ of cells; $1,5-25 \%$; $2,26-50 \%$; $3,51-75 \%$; and 4 , $76 \sim 100 \%)$. Histoscore was defined as the sum of the intensity and distribution scores. For interpretation of the immunohistochemical stain results for $\gamma$-H2AX, dark brown stained nuclei were counted as being positive for antibody, and labeling indices were determined as follows: (number of positive hepatocytic nuclei in five randomly selected fields at $\times 400$ magnification)/(total number of hepatocytic nuclei) $\times 100 \%$.

Telomere terminal restriction fragment length analysis Telomere terminal restriction fragments were measured as previously described [2]. Briefly, telomere length was measured by Southern blotting. Two $\mu \mathrm{g}$ of digested DNA was separated on $0.7 \%$ agarose gel. Hybridization was 
carried out with 3 ' -end DIG-labeled d(TTAGGG) ${ }_{4}$ (Roche Molecular Biochemicals, Mannheim, Germany) and detected as recommended by the manufacturer. The resulting X-ray film was scanned with a luminescent image analyzer (Fujifilm, Tokyo, Japan), and the telomere signals in each lane were quantified as a grid object, defined as a single column with 25 rows, using Image Gauge Software 2.54 (Fujifilm).

\section{Telomere fluorescent in situ hybridization (FISH) combined immunostaining for $\mathrm{Y}-\mathrm{H} 2 \mathrm{AX}$}

TIF were detected in representative sections of formalinfixed paraffin-embedded tissues by staining for $\gamma$-H2AX and by telomere in situ hybridization using a telomerespecific peptide nucleic acid probe $\left(\mathrm{Cy} 3-(\mathrm{CCCTAA})_{3}\right.$; Panagene, Daejeon, Korea). Tissue sections were deparaffinized and then rehydrated with graded alcohol. After incubation in $0.2 \mathrm{~N} \mathrm{HCl}$ for $20 \mathrm{~min}$, slides were boiled in $10 \mathrm{mM}$ citrate buffer, $\mathrm{pH} 6.0$, for $15 \mathrm{~min}$ to retrieve antigens. Sections were then treated with protease solution (Abbott Molecular Inc, Des Plaines, IL, USA) and fixed. Sections were dehydrated in $100 \%$ ethanol for 5 min. After air-drying, slides were applied $10 \mu \mathrm{L}$ of a telomere peptide nucleic acid probe mixture (70\% formamide deionized, $5 \mathrm{mM}$ Tris- $\mathrm{HCl}, \mathrm{pH}$ 7.4, $1 \mathrm{mM} \mathrm{MgCl}_{2}$, $0.45 \mathrm{mM}$ Citric acid, $4.1 \mathrm{mM} \mathrm{NaHPO}, 0.1 \mu \mathrm{g}$ telomerespecific peptide nucleic acid probe, $5 \%$ blocking reagent [Roche Molecular Biochemicals]), denatured at $80^{\circ} \mathrm{C}$ for $3 \mathrm{~min}$, and hybridized at $30^{\circ} \mathrm{C}$ for $2 \mathrm{hrs}$. Slides were then washed sequentially with $0.6 \mathrm{X} \mathrm{SSC} / 70 \%$ formamide (90 mM NaCl, 9 mM Na-citrate [pH 7.0]; $3 \times 15$ min), $2 \mathrm{X}$ SSC $(2 \times 15 \mathrm{~min})$, PBS $(1 \times 5 \mathrm{~min})$, and PBST $(\mathrm{PBS}+0.1 \%$ Tween 20:15 min), and then blocked with 5\% BSA for 5 min. Immunostaining using primary polyclonal anti- $\gamma-$ H2AX (1:500; Novus Biologicals, Littleton, CO, USA) and secondary Alexa Fluor 488-conjugated goat anti rabbit antibody (1:1,000; Invitrogen) was performed.

\section{Statistical analysis}

Statistical analysis was conducted using SPSS (version 18.0.0; SPSS Inc., Chicago, IL, USA) and R package software (version 3.0.2; http://www.R-project.org), applying the Mann-Whitney test, Spearman's correlation coefficient, linear model, and log linear model as deemed appropriate. Significance was set at $P<0.05$ for all tests.

\section{Results}

\section{Stathmin expression in HBV-related multistep} hepatocarcinogenesis

The mRNA levels of stathmin were $0.3 \pm 0.12($ mean \pm SD) (range, $0.2 \sim 0.5)$ in normal liver, $0.5 \pm 0.61(0.1 \sim 2.1)$ in liver cirrhosis, $0.8 \pm 0.57(0.1 \sim 2.0)$ in low-grade DNs, $1.5 \pm 1.29(0.1 \sim 4.7)$ in high-grade DNs, and $2.3 \pm 1.95$ $(0.4 \sim 7.0)$ in HCC. Stathmin mRNA levels gradually increased as multistep hepatocarcinogenesis progressed from normal liver, low-grade DNs, and high-grade DNs to HCC, which showed the highest level of expression and statistical significance $(P$ for trend $<0.001$ ) (Figure $1 \mathrm{~A}$ ). The differences in mRNA levels were statistically significant between normal liver and high-grade DNs, as well as between liver cirrhosis and high-grade DNs (all, $P<0.05$ ). Stathmin mRNA levels in HCC were significantly higher than those in normal liver, liver cirrhosis, and low-grade DNs (all, $P<0.05$ ).

Similarly, the expression of stathmin protein also gradually increased as multistep hepatocarcinogenesis progressed towards HCC ( $P$ for trend $<0.001$ ) (Figure $1 \mathrm{~B}$, Figure $2 \mathrm{~B}$ ). Most normal liver specimens showed no expression of stathmin protein, except for one case that showed weak expression of histoscore grade 1 . The mean histoscores for stathmin were $1.3 \pm 0.63$ in liver cirrhosis, $2.0 \pm 1.57$ in low-grade DNs, $2.6 \pm 2.12$ in high-grade DNs, and $4.1 \pm 1.77$ in HCC. Stathmin histoscores were significantly different between liver cirrhosis and HCC, as well as between low-grade DNs and HCC; moreover, stathmin histoscores were significantly greater in liver cirrhosis, low-grade DNs, high-grade DNs, and HCC than in normal liver (all, $P<0.05$ ).

\section{EF1a expression in HBV-related multistep hepatocarcinogenesis}

The mRNA levels of EF1 $\alpha$ were $2.9 \pm 0.71$ (mean \pm SD) (range, $2.3 \sim 4.0)$ in normal liver, $1.9 \pm 0.83(0.2 \sim 3.2)$ in liver cirrhosis, $1.6 \pm 0.74(0.3 \sim 3.1)$ in low-grade DNs, $2.5 \pm 1.76(0.9 \sim 8)$ in high-grade $\mathrm{DNs}$, and $3.9 \pm 3.19$ $(1.1 \sim 13.2)$ in HCC (Figure 1C). HCC showed the highest level of EF1 $\alpha$ mRNA expression, which was significantly higher than that in low-grade DNs $(P=0.009)$, whereas liver cirrhosis and low-grade DNs showed lower levels of EF1 $\alpha$ mRNA expression than normal liver $(P<0.05$ for both). The mRNA expression of EF1 $\alpha$ showed significant increases as multistep hepatocarcinogenesis progressed towards HCC ( $P$ for trend $=0.035)$.

$E F 1 \alpha$ protein was not expressed in normal liver, but was detected in dysplastic and HCC cells (Figure 2C). The histoscores of EF1 $\alpha$ protein were $0.2 \pm 0.55$ (mean \pm SD) (range, $0 \sim 2$ ) in liver cirrhosis, $0.7 \pm 1.14(0 \sim 4)$ in low-grade DNs, $1.3 \pm 1.36(0 \sim 5)$ in high-grade DNs, and $2.4 \pm 1.87(1 \sim 7)$ in HCC (Figure 1D), and gradually increased from liver cirrhosis, low-grade DNs, highgrade $\mathrm{DNs}$ to $\mathrm{HCC}$ with statistical significance $(P$ for trend $<0.001)$. HCC showed the highest EF1 $\alpha$ histoscore, which was significantly higher than that for low-grade DNs, liver cirrhosis and normal liver (all, $P<0.05$ ). EF1 $\alpha$ histoscores were also significantly greater in high-grade DNs than in liver cirrhosis $(P=0.002)$ and normal liver $(P=0.013)$. The histoscores of EF1 $\alpha$ and stathmin were shown to be positively correlated $(P<0.001, \mathrm{R}=0.443)$, 


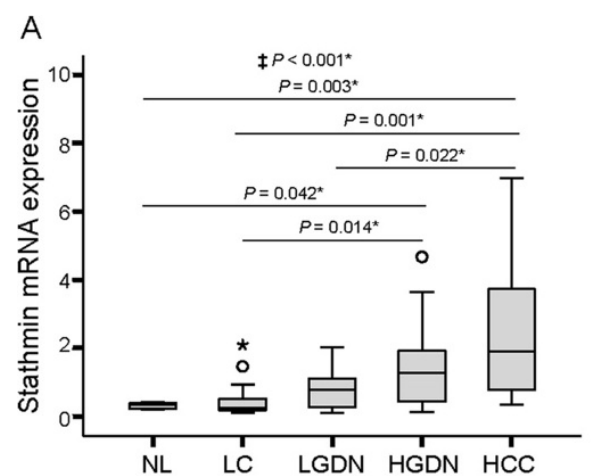

C

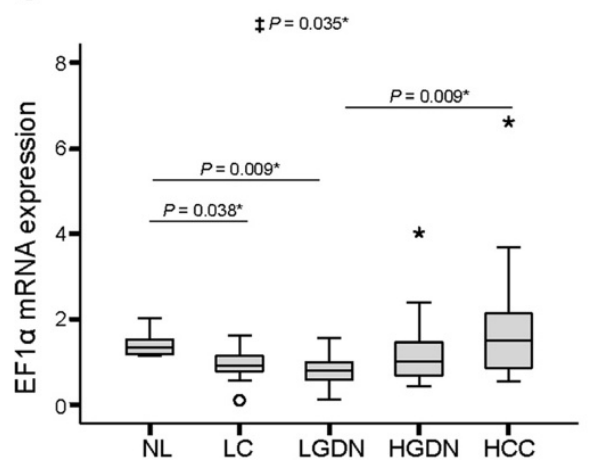

B

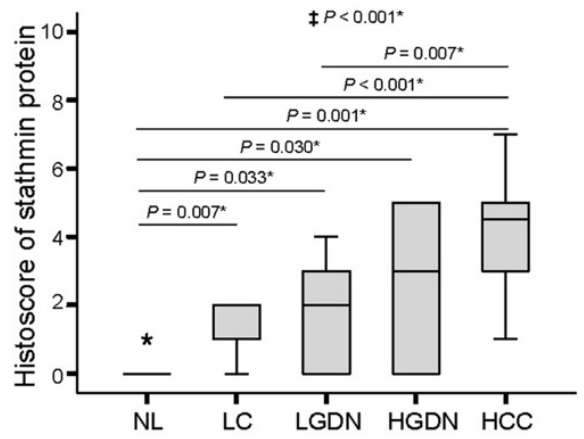

D

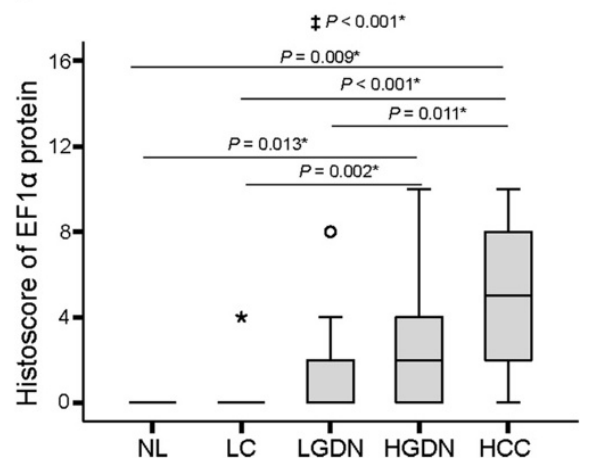

Figure 1 Expression levels of stathmin and elongation factor 1a (EF1a) in HBV-related multistep hepatocarcinogenesis. Box plots show expression levels of stathmin mRNA (A), stathmin protein (B), EF1 a mRNA (C), and EF1a protein (D). ‡ Statistical significance (linear trend model, $P<0.05)$ *Statistical significance $(P<0.05)$. NL, normal liver; LC, liver cirrhosis; LGDN, low-grade dysplastic nodules; HGDN, high-grade dysplastic nodules; HCC, hepatocellular carcinoma.

whereas EF1 $\alpha$ and stathmin mRNA levels showed no significant correlation $(P=0.558, \mathrm{R}=0.075)$.

Telomere length and its relationship with stathmin and EF1a expression in HBV-related multistep hepatocarcinogenesis

Telomere length gradually decreased as multistep hepatocarcinogenesis progressed towards HCC, with statistical significance $(P$ for trend $=0.032)$; part of this data was previously reported [2]. Telomere lengths were $8.5 \pm 0.92$ (mean \pm SD) (range, $7.3 \sim 9.3$ ) in normal liver, $7.6 \pm 1.81$ (4.6 11.3) in liver cirrhosis, $6.4 \pm 1.63(4.7 \sim 10.2)$ in lowgrade DNs, $6.8 \pm 2.54(2.9 \sim 11.5)$ in high-grade DNs, and $6.3 \pm 2.19(3.4 \sim 10.7)$ in HCC (Figure 3A).

Next, the associations of telomere length with stathmin and EF1 $\alpha$ expression were evaluated. Both mRNA levels and histoscores for stathmin showed significant negative correlations with telomere length (Figure 3B and $C)(P=0.004, \mathrm{R}=-0.353$ and $P=0.029, \mathrm{R}=-0.276$, respectively). EF1 $\alpha$ histoscore showed a significant negative correlation with telomere length $(P=0.001, \mathrm{R}=-0.404)$ (Figure 3D), whereas EF1 $\alpha$ mRNA level showed no significant correlation with telomere length $(P=0.719, \mathrm{R}=-0.046)$ (Additional file 2: Figure S1A).
$\mathrm{Y}$-H2AX expression and its relationship with stathmin and EF1a expression and telomere length in HBV-related multistep hepatocarcinogenesis

To evaluate DNA damage in multistep hepatocarcinogenesis, immunohistochemical analysis of $\gamma-\mathrm{H} 2 \mathrm{AX}$ was performed. The expression of $\gamma-\mathrm{H} 2 \mathrm{AX}$ protein was detected in the nuclei of dysplastic and neoplastic cells. The $\gamma$ $\mathrm{H} 2 \mathrm{AX}$ labeling indices were $1.3 \pm 4.13$ (mean $\pm \mathrm{SD}$ ) (range, $0 \sim 15.0)$ in liver cirrhosis, $2.4 \pm 5.67(0 \sim 21.3)$ in lowgrade DNs, $4.1 \pm 10.42(0 \sim 40.2)$ in high-grade DNs, and $10.3 \pm 17.00(0 \sim 49.0)$ in HCC (Figure 4A). $\gamma$-H2AX labeling index showed a gradual increase from liver cirrhosis, low-grade DNs, and high-grade DNs to HCC with statistical significance $(P$ for trend $=0.016) \cdot \gamma$-H2AX labeling index in HCC was significantly higher than that in normal liver, liver cirrhosis, low-grade DNs, and high-grade DNs (all, $P<0.05$ ). Additionally, $\gamma$-H2AX labeling index showed a significant negative correlation with telomere length $(P=0.018, \mathrm{R}=-0.300)$; higher expression of $\gamma-\mathrm{H} 2 \mathrm{AX}$ labeling index was found in lesions with shorter telomere lengths (Figure 4B).

The associations between $\gamma$-H2AX labeling index and the expression levels of stathmin and $E F 1 \alpha$ were further analyzed. $\gamma-\mathrm{H} 2 \mathrm{AX}$ labeling index showed a significant positive correlation with both mRNA levels and histoscores of 


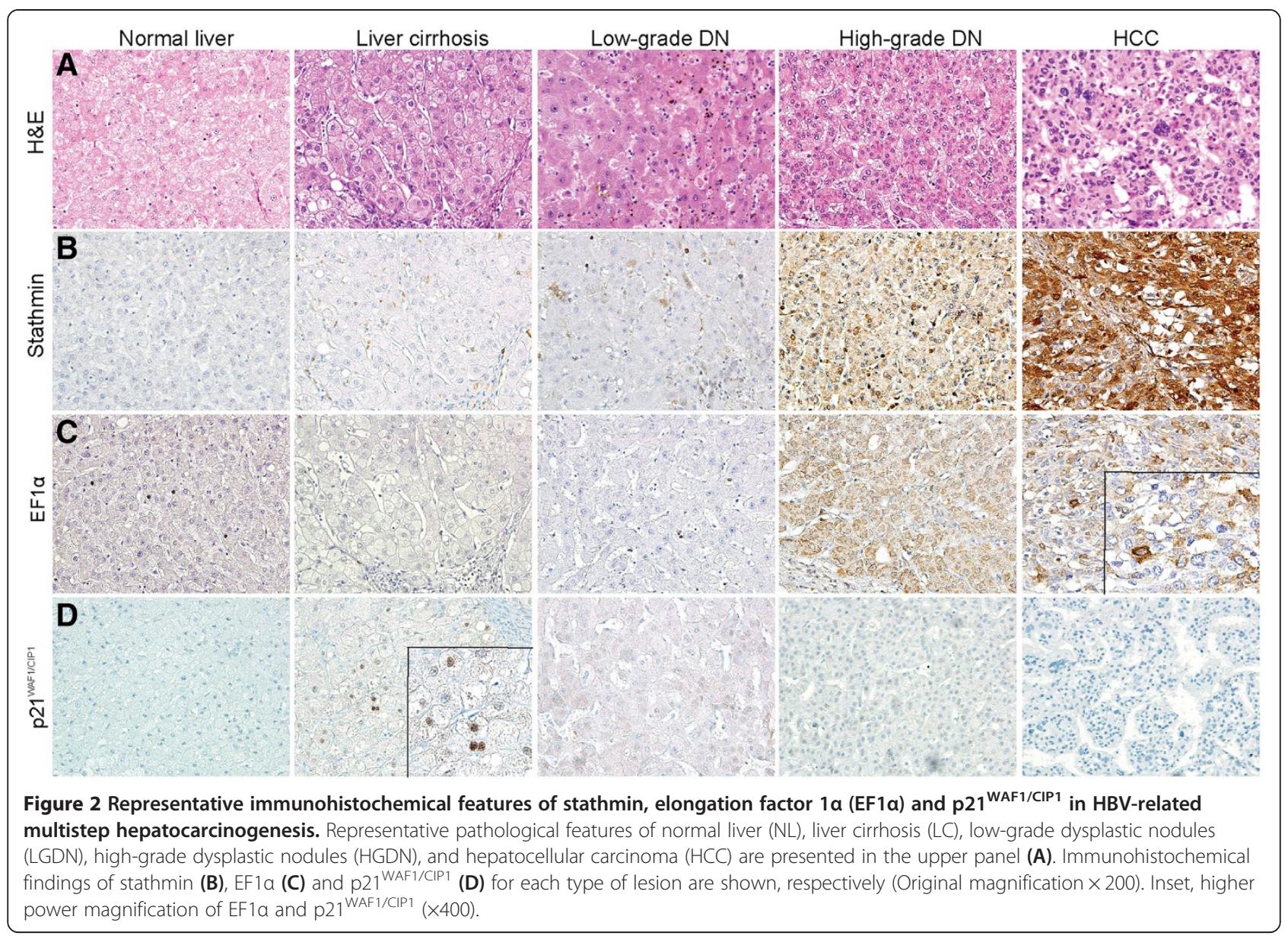

stathmin $(P<0.001, \mathrm{R}=0.456$ and $P=0.005, \mathrm{R}=0.351$, respectively), indicating that an increase in stathmin expression is associated with DNA damage (Figure $4 \mathrm{C}$ and D). For EF1 $\alpha$ expression, histoscores of EF1 $\alpha$ protein showed a positive correlation with $\gamma-\mathrm{H} 2 \mathrm{AX}$ index $(P<$ $0.003, R=0.373$ ) (Figure 4D); however, we observed no significant correlations between EF1 $\alpha$ mRNA level and $\gamma$ H2AX labeling index $(P=0.555, \mathrm{R}=-0.076)$ (Additional file 2: Figure $\mathrm{S} 1 \mathrm{~B})$.

\section{Telomere dysfunction induced foci (TIF) and their relationship with stathmin and EF1a expression in HBV-related multistep hepatocarcinogenesis}

In order to determine the fractions of TIF during human multistep hepatocarcinogenesis, we performed telomere FISH combined with immunostaining for anti- $\gamma-\mathrm{H} 2 \mathrm{AX}$ to detect colocalization of telomeres and $\gamma-\mathrm{H} 2 \mathrm{AX}$ foci (Figure 5A). A gradual increase of TIF was observed as multistep hepatocarcinogenesis progressed towards HCC with statistical significance $(P$ for trend $<0.01)$ (Figure $5 \mathrm{~B})$. Normal liver showed the lowest average number of TIF, 0.1 \pm 0.30 (mean $\pm \mathrm{SD}$ ) (range, $0.06 \sim 0.16$ ), and the number of TIF gradually increased as multistep hepatocarcinogenesis progressed from normal liver, liver cirrhosis, low-grade DNs, and high-grade DNs to HCC (liver cirrhosis; $0.1 \pm 0.06$ [0.06 0.21], low-grade DNs; $0.3 \pm 0.23$ [0.06 0.69], highgrade DNs; $0.4 \pm 0.12$ [0.26 0.76], HCC; $0.8 \pm 0.48$ [0.24 1.56]). HCC showed a significantly higher number of TIF, compared to normal liver, liver cirrhosis, and low-grade DNs (all, $P<0.05$ ); high-grade DNs showed a significantly higher number of TIF, compared to normal liver and liver cirrhosis (all, $P<0.05$ ); and low-grade DNs showed a significantly higher number of TIF than normal liver $(P=0.048)$. We further analyzed the relationships between TIF and telomere length, as well as TIF and the protein expression levels of stathmin and EF1 $\alpha$. TIF showed a significant negative correlation with telomere length $(P=$ $0.020, R=-0.415$, Figure $5 C$ ). $E F 1 \alpha$ histoscore showed a significant positive correlation with TIF, suggesting that higher EF1 $\alpha$ expression is associated with a greater number of TIF, indicating increased telomere dysfunction $(P<0.001, \mathrm{R}=0.602)$ (Figure $5 \mathrm{D})$. Stathmin histoscore, however, showed no significant correlation with TIF $(P=0.326, \mathrm{R}=0.182)$ (Additional file 2: Figure S1C). We also observed no significant correlations between TIF and the mRNA levels of both stathmin and 
A

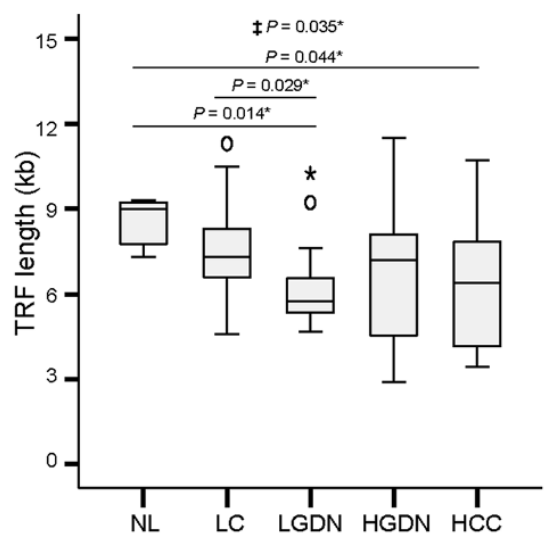

C

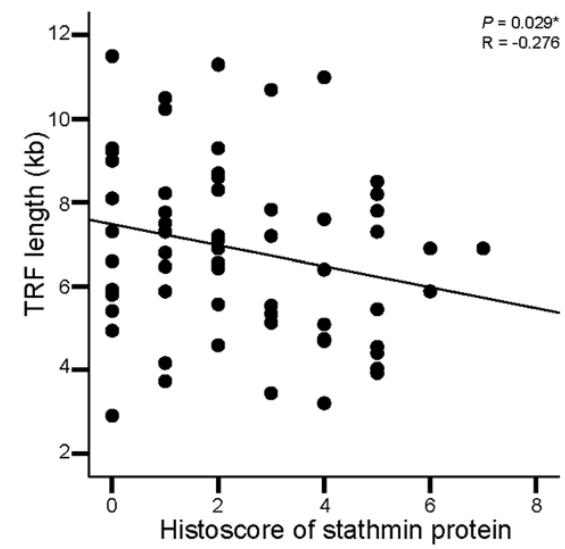

B

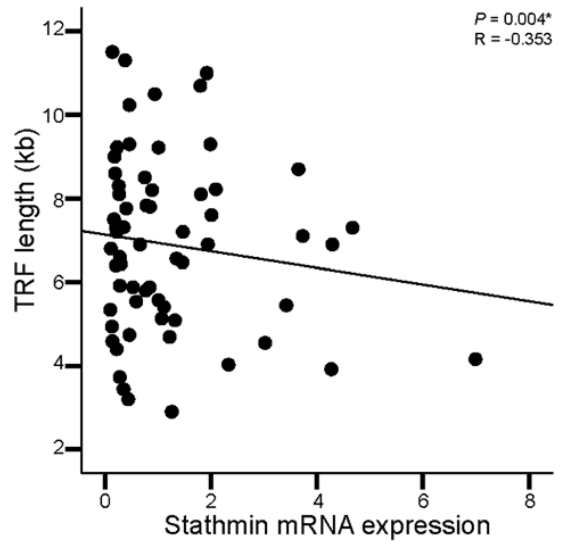

D

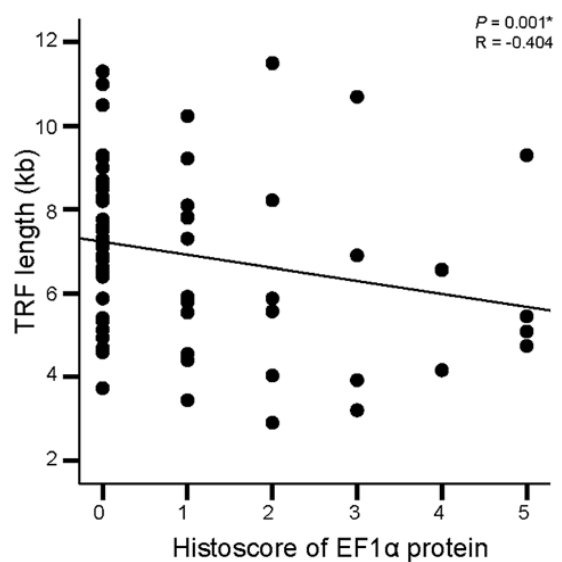

Figure 3 Telomere length and the correlations thereof with stathmin and elongation factor 1a (EF1a) expression in HBV-related multistep hepatocarcinogenesis. A. Telomere terminal restriction fragment (TRF) length in normal liver (NL), liver cirrhosis (LC), low-grade dysplastic nodules (LGDN), high-grade dysplastic nodules (HGDN), and hepatocellular carcinoma (HCC). B-C. Correlation between TRF length and stathmin mRNA (B) and stathmin protein levels (C). D. Correlation between TRF length and EF1a protein levels. \# Statistical significance (linear trend model, $P<0.05)$. *Statistical significance $(P<0.05)$.

EF1 $\alpha \quad(P=0.360, \quad \mathrm{R}=0.170$ and $P=0.263, \quad \mathrm{R}=0.205$, respectively).

p21 ${ }^{\text {WAF1/CIP1 }}$ labeling index and its relationship with TIF, and stathmin and EF1a expression in HBV-related multistep hepatocarcinogenesis

The expression of $\mathrm{p} 21^{\mathrm{WAF} 1 / \mathrm{CIP} 1}$ protein was investigated in multistep hepatocarcinogenesis; part of the data was previously reported [2]. The p $21^{\mathrm{WAF} 1 / \mathrm{CIP} 1}$ labeling indices showed gradual statistically significant decreases as multistep hepatocarcinogenesis progressed towards HCC ( $P$ for trend $=0.002$ ) (Figure $2 \mathrm{D}$ and Figure 6A).

The p21 ${ }^{\text {WAF1/CIP1 }}$ labeling indices were $10.4 \pm 6.09$ (mean \pm SD) (range, $3.1 \sim 18.6$ ) in normal liver, $24.9 \pm$ $13.55(1.6 \sim 47.2)$ in liver cirrhosis, $7.9 \pm 2.90(2.7 \sim 11.2)$ in low-grade DNs, $11.0 \pm 11.11(0.8 \sim 44.7)$ in high-grade DNs, and $5.9 \pm 8.08(0.4 \sim 30.2)$ in HCC. p $21^{\text {WAF1/CIP1 }}$ labeling index was highest in liver cirrhosis, which was significantly higher than that in normal liver, low-grade DNs, high-grade DNs, and HCC (all, $P<0.05$ ). The p21 ${ }^{\text {WAF1/CIP1 }}$ labeling index of HCC was the lowest, and was significantly lower than that in low-grade DNs, high-grade DNs, and liver cirrhosis (all, $P<0.05$ ).

p21 WAF1/CIP1 labeling index showed a significant negative correlation with $\operatorname{TIF}(P=0.045, \mathrm{R}=-0.362)$ (i.e., higher TIF was correlated with lower p $21^{\mathrm{WAF} 1 / \mathrm{CIP} 1} 1 \mathrm{la}$ beling index) (Figure 6B). Additionally, associations between $\mathrm{p} 21^{\mathrm{WAF} 1 / \mathrm{CIP} 1}$ labeling index and the expression levels of stathmin and EF1 $\alpha$ were analyzed. Both stathmin mRNA levels and histoscores showed a negative correlation with $\mathrm{p} 21^{\mathrm{WAF} 1 / \mathrm{CIP} 1}$ labeling index (Figure $6 \mathrm{C}$ and D) $(P=0.008, \mathrm{R}=-0.333$, and $P=0.05, \mathrm{R}=-0.248$, respectively) (i.e., higher stathmin expression was correlated with lower $\mathrm{p} 21^{\mathrm{WAF} 1 / \mathrm{CIP} 1}$ labeling index). EF1 $\alpha$ histoscore showed a significant negative correlation with $\mathrm{p} 21^{\mathrm{WAF} 1 / \mathrm{CIP} 1}$ labeling index, as higher EF1 $\alpha$ histoscores 


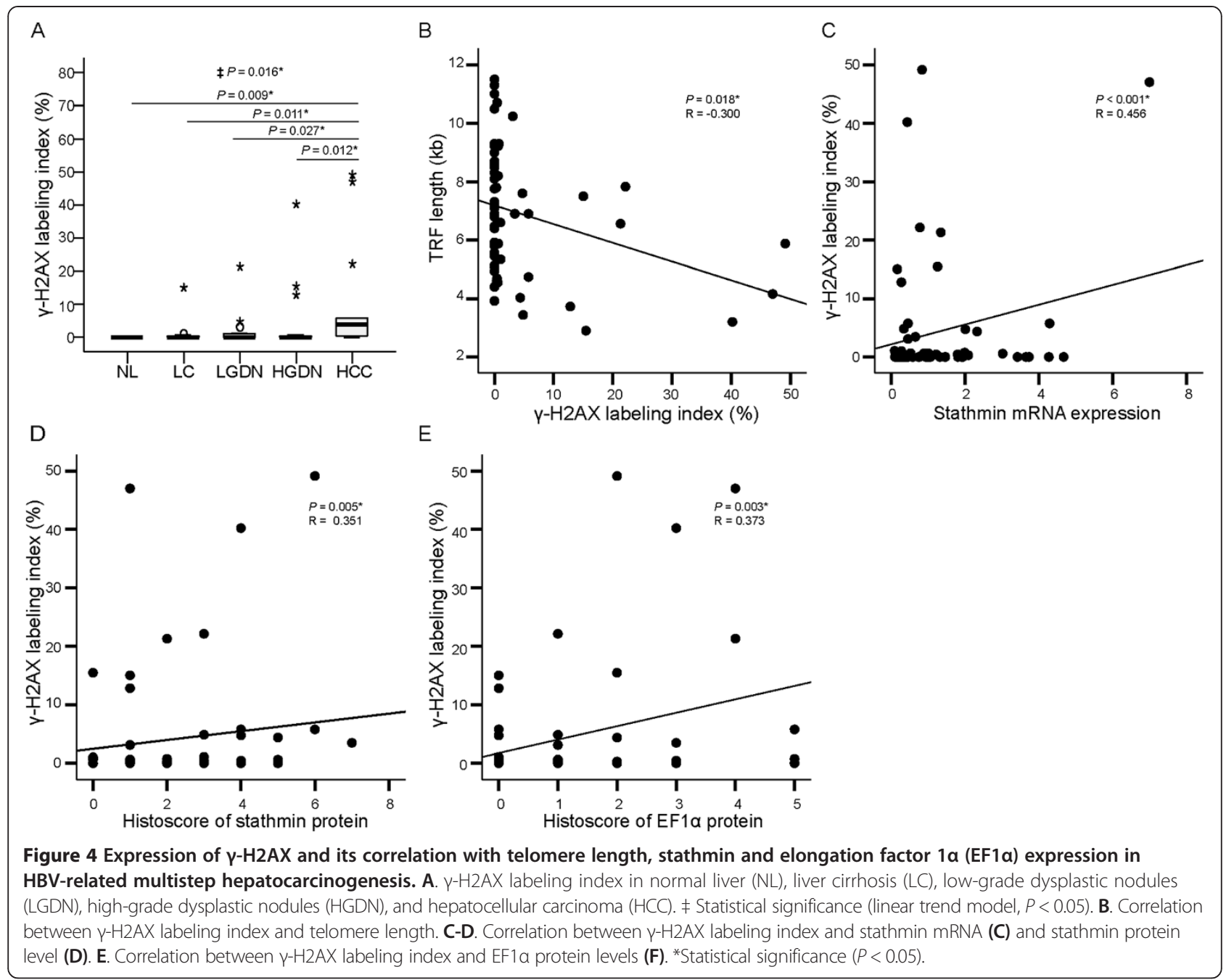

were correlated with lower $\mathrm{p} 21^{\mathrm{WAF} 1 / \mathrm{CIP1}}$ labeling index $(P<0.001, \quad \mathrm{R}=-0.486)$, whereas EF1 $\alpha$ mRNA levels showed no significant correlation with $\mathrm{p} 21^{\mathrm{WAF} 1 / \mathrm{CIP} 1} \mathrm{la}$ beling index $(P=0.383, \mathrm{R}=-0.112)$ (Figure $6 \mathrm{E}$ and Additional file 2: Figure S1D).

\section{Discussion}

Telomere erosion occurs in proliferating cells with insufficient telomerase activity; thereby, critically short telomeres become dysfunctional and trigger apoptosis and/or senescence as a tumor suppressive mechanism. Meanwhile, mutations that impair the DNA damage response pathways allow for survival of cells with critically short telomeres. As a result, cells with dicentric chromosomes can proliferate and lead to the accumulation of genomic instability due to breakage-fusion-bridge cycles. In the present study, a gradual increase in shortened and dysfunctional telomeres was found as HBV-related multistep hepatocarcinogenesis progressed to HCC. Low-grade DNs showed a significantly higher number of TIF than normal liver; high-grade DNs exhibited a significantly greater number of TIF than liver cirrhosis and normal liver; and HCC showed the highest number of dysfunctional telomeres. Additionally, telomere length was inversely correlated with $\gamma$-H2AX labeling index, a DNA damage marker. These findings support that telomere dysfunction and DNA damage are important in the progression of HBV-related multistep hepatocarcinogenesis.

Stathmin was recently shown to be induced in response to telomere dysfunction and DNA damage in human aging and diseases [6]. Excessive stathmin activity was reported to generate chromosomal instability through blockage, subsequent to metaphase-to-anaphase transition, by decreasing the fidelity of chromosome segregation to spindle poles during anaphase in an in vitro study [21]. In the present study, stathmin expression at both the mRNA and protein level increased gradually as multistep hepatocarcinogenesis progressed from liver cirrhosis, lowgrade DNs, and high-grade DNs to $\mathrm{HCC}$, which showed the highest levels of expression. Interestingly, stathmin 


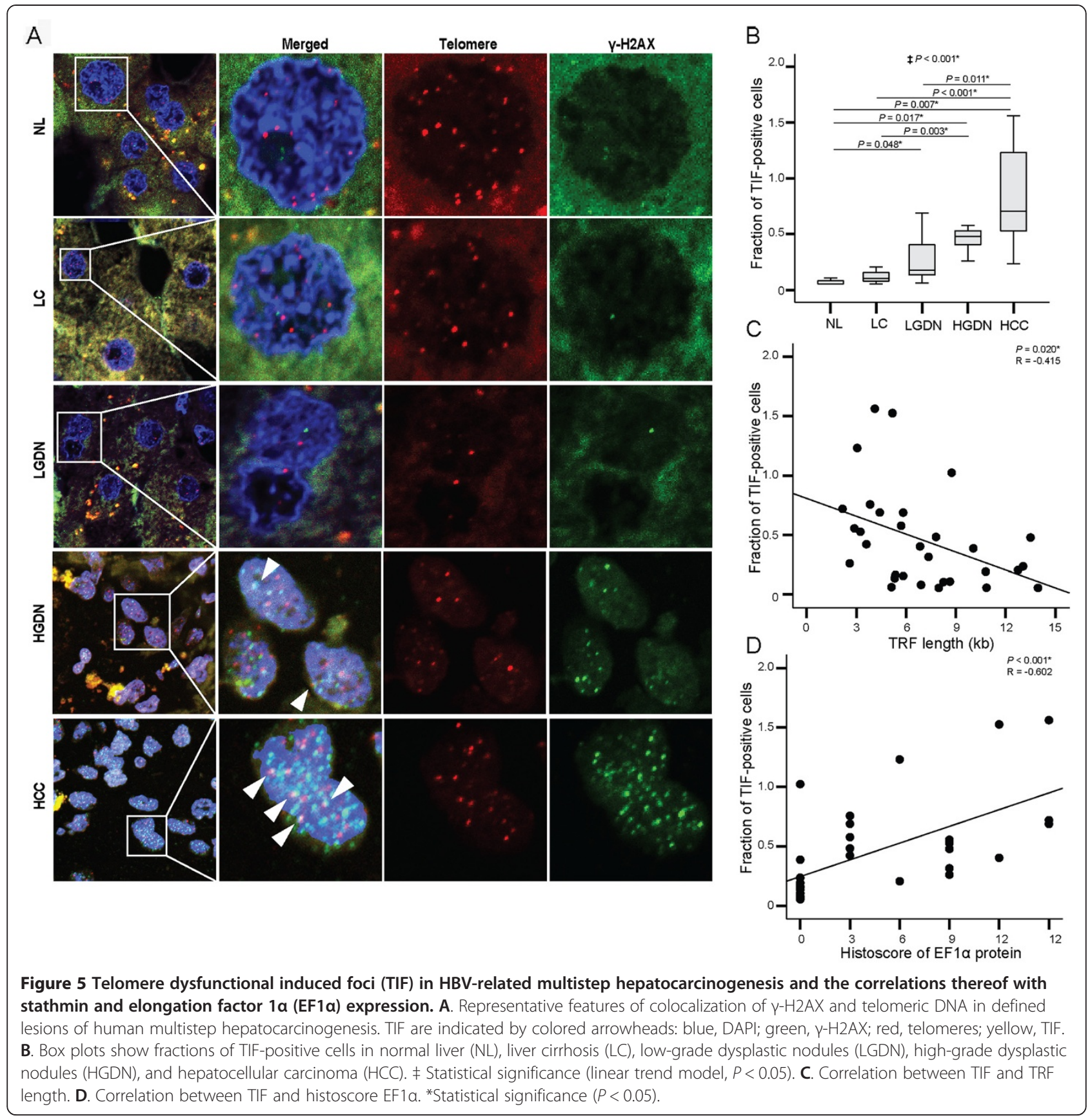

mRNA levels were significantly higher in high-grade DNs than in liver cirrhosis and normal liver and the histoscores of stathmin protein were higher in high-grade DNs and low-grade DNs than normal liver; stathmin expression was not detected in normal liver. Additionally, the protein and mRNA expression levels of stathmin were well correlated with $\gamma$-H2AX labeling index and telomere shortening; moreover, fractions of TIF-positive cells were also well correlated with telomere length shortening.

The primary function of EF $1 \alpha$ is to transport aminoacyltRNA to ribosomes during protein translation. The overexpression of EF1 $\alpha$ was previously reported to be associated with cell proliferation, oncogenic transformation, and delayed cell senescence in human and rodent cells $[16,17]$. As well, previous functional studies demonstrated that EF1 $\alpha$ regulates the proliferation, survival, and motility of breast cancer cells [18] and induces higher proliferation capacity in undifferentiated HCC cell lines [19]. In the present study, EF1 $\alpha$ protein expression gradually increased in human multistep hepatocarcinogenesis, and was highest in HCC. Interestingly, EF1 $\alpha$ histoscore in HGDN was significantly higher than that in normal liver 


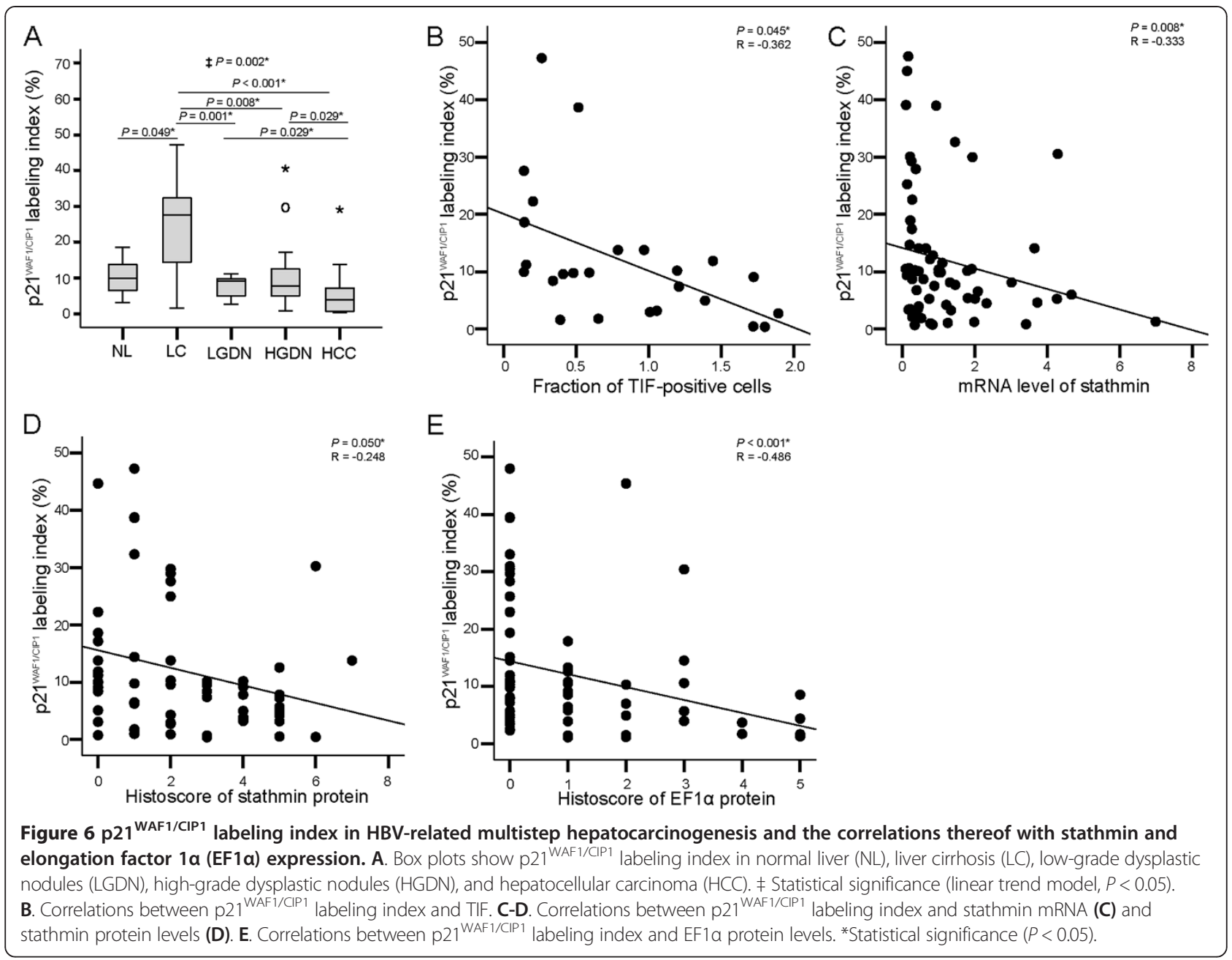

and liver cirrhosis. The expression levels of EF1 $\alpha$ protein were well correlated with $\gamma$-H2AX labeling index and TIF, whereas they exhibited a significant inverse correlation with telomere length. Meanwhile, EF1 $\alpha$ mRNA level showed no significant correlation with telomere length, $\gamma$ H2AX labeling index, TIF, or p $21^{\mathrm{WAF} 1 / \mathrm{CIP} 1}$ labeling index, and further investigation of a translational regulation mechanism for EF1 $\alpha$ is needed. Notwithstanding, our data suggested that the expression of stathmin and EF1 $\alpha$ arises in DNs as precancerous lesions and gradually increases along with progression of human hepatocarcinogenesis, suggesting that expression of stathmin and EF1 $\alpha$ is induced by shortened and dysfunctional telomeres in B viral multistep hepatocarcinogenesis.

$\mathrm{p} 21^{\mathrm{WAF} 1 / \mathrm{CIP} 1}$ is a potent cyclin-dependent kinase inhibitor and the expression of this gene is controlled by the tumor suppressor protein $\mathrm{p} 53$, through which this protein mediates the p53-dependent cell cycle G1 phase arrest in response to a variety of stress stimuli [22]. In this study, p21 ${ }^{\text {WAF1/CIP1 }}$ was inactivated in low-grade DNs, high-grade DNs, and HCC, in contrast to its high expression in liver cirrhosis. Interestingly, $\mathrm{p} 21^{\mathrm{WAF} 1 / \mathrm{CIP} 1}$ labeling index showed a significant negative correlation with TIF, stathmin mRNA level, and the histoscores of EF1 $\alpha$ protein in this study. Accordingly, the p $21^{\mathrm{WAF} 1 / \mathrm{CIP} 1}$ cell cycle check point was discerned to be a defense mechanism against damaged and transformed cells, triggered by dysfunctional telomeres in multistep hepatocarcinogenesis.

The natural history of DNs has not been fully clarified, and prospective studies conducted in large series of histologically proven non-neoplastic nodules detected by ultrasonography during surveillance programs of cirrhosis have demonstrated a wide evolutionary fate for such lesions. The hazard ratios of high-grade DNs, low-grade DNs, and large regenerative nodules for transformation to HCC were reported to be $16.8,2.96$, and 1.0 , respectively $[4,5]$. However, it is difficult to predict the biological behavior of individual hepatic nodules, as some high-grade DNs remain stable for a long time period and a few of them even disappear. Therefore, the assessment of shortened and dysfunctional telomeres may be helpful to evaluating biological characteristics of hepatic 
nodules, although direct measurement of telomere dysfunction using telomere FISH combined immunostaining is very time consuming and labor intensive. Nevertheless, this study revealed that the expressions of stathmin and EF1 $\alpha$ are good indicators of shortened and dysfunctional telomeres, and the evaluation of the expression levels thereof in conjunction with $\mathrm{p} 21^{\mathrm{WAF} 1 / \mathrm{CIP} 1}$ may prove helpful in characterizing the biological nature of hepatic nodules in B viral cirrhotic patients.

Here, we demonstrated for the first time that the expression of stathmin and EF1 $\alpha$ are closely related to telomere dysfunction, DNA damage, and inactivation of $\mathrm{p} 21^{\mathrm{WAF} 1 / \mathrm{CIP} 1}$ in the defined lesions of HBV-related multistep hepatocarcinogenesis, including liver cirrhosis, low-grade DNs, high-grade DNs, and HCC. Accordingly, assessment of stathmin and EF1 $\alpha$ levels as a reflection of telomere dysfunction may be helpful in evaluating the biological characteristics of precancerous hepatic nodules in hepatitis B viral cirrhotic patients.

\section{Additional files}

Additional file 1: Table S1. Antibodies used in this study.

Additional file 2: Figure S1. Correlation of stathmin and elongation factor 1a (EF1a) expression with telomere dysfunction and DNA damage in HBV-related multistep hepatocarcinogenesis. A-B. Scatter plots reveal a correlation between EF1 a mRNA expression and telomere terminal restriction fragment (TRF) length (A) and $\mathrm{\gamma}-\mathrm{H} 2 \mathrm{AX}$ labeling index (B). C. Correlation between telomere dysfunction induced foci (TIF) and stathmin protein level. D. Scatter plot of a correlation between p21 WAF1/CIP1 labeling index and EF1a mRNA expression.

\section{Competing interests}

All authors have no competing interest to disclose.

\section{Author's contributions}

EY Ahn and JE Yoo designed the study and carried out experiments and analysis of data and drafted manuscript. J Choi analyzed some part of the data and participated in collection of human specimens. H Rhee, MS Kim, JE Ko and JS Lee participated in collection of human specimens. YN Park conceived of the study, participated in its design and coordination, and helped to draft the manuscript. All authors read and approved the final manuscript.

\section{Acknowledgements}

This study was supported by a grant from the Korea Health Technology R\&D Project through the Korea Health Industry Development Institute (KHIDI), funded by the Ministry of Health \& Welfare, Republic of Korea (grant number: HI12C0555)

We would like to thank Anthony Milliken for his help with English editing. We would also like to thank the Biostatistics Collaboration Unit, Yonsei University College of Medicine, for their statistical assistance and Yonsei-Carl Zeiss Advanced Imaging Center, Yonsei University College of Medicine, for their technical assistance.

\section{Author details}

${ }^{1}$ Department of Pathology, Yonsei University College of Medicine, 250 Seongsan-ro, Seodaemun-gu, Seoul, South Korea. ${ }^{2}$ Brain Korea 21 PLUS Project for Medical Science, Seoul, South Korea. ${ }^{3}$ Department of Pathology, Wonju Christian Hospital, Wonju, South Korea. ${ }^{4}$ Department of Surgery, Yonsei University College of Medicine, Seoul, South Korea. ${ }^{5}$ Severance Biomedical Science Institute, Yonsei University College of Medicine, Seoul,
South Korea. ${ }^{6}$ Integrated Genomic Research Center for Metabolic Regulation, Yonsei University College of Medicine, Seoul, South Korea.

Received: 11 February 2014 Accepted: 27 May 2014

Published: 31 May 2014

\section{References}

1. Parkin DM, Bray F, Ferlay J, Pisani P: Global cancer statistics, 2002. CA Cancer J Clin 2005, 55:74-108.

2. Lee YH, Oh BK, Yoo JE, Yoon SM, Choi J, Kim KS, Park YN: Chromosomal instability, telomere shortening, and inactivation of p21(WAF1/CIP1) in dysplastic nodules of hepatitis B virus-associated multistep hepatocarcinogenesis. Mod Pathol 2009, 22:1121-1131.

3. International Consensus Group for Hepatocellular Neoplasia: Pathologic diagnosis of early hepatocellular carcinoma: a report of the international consensus group for hepatocellular neoplasia. Hepatology 2009, 49:658-664.

4. Borzio M, Fargion S, Borzio F, Fracanzani AL, Croce AM, Stroffolini T, Oldani $\mathrm{S}$, Cotichini R, Roncalli M: Impact of large regenerative, low grade and high grade dysplastic nodules in hepatocellular carcinoma development. J Hepatol 2003, 39:208-214.

5. Kobayashi M, Ikeda K, Hosaka T, Sezaki H, Someya T, Akuta N, Suzuki F, Suzuki Y, Saitoh S, Arase Y, Kumada H: Dysplastic nodules frequently develop into hepatocellular carcinoma in patients with chronic viral hepatitis and cirrhosis. Cancer 2006, 106:636-647.

6. Jiang H, Schiffer E, Song Z, Wang J, Zurbig P, Thedieck K, Moes S, Bantel H, Saal N, Jantos J, Brecht M, Jeno P, Hall MN, Hager K, Manns MP, Hecker H, Ganser A, Dohner K, Bartke A, Meissner C, Mischak H, Ju Z, Rudolph KL: Proteins induced by telomere dysfunction and DNA damage represent biomarkers of human aging and disease. Proc Natl Acad Sci U S A 2008, 105:11299-11304.

7. Rubin $\mathrm{Cl}$, Atweh GF: The role of stathmin in the regulation of the cell cycle. J Cell Biochem 2004, 93:242-250.

8. Charbaut E, Curmi PA, Ozon S, Lachkar S, Redeker V, Sobel A: Stathmin family proteins display specific molecular and tubulin binding properties. J Biol Chem 2001, 276:16146-16154.

9. Curmi PA, Gavet O, Charbaut E, Ozon S, Lachkar-Colmerauer S, Manceau V, Siavoshian S, Maucuer A, Sobel A: Stathmin and its phosphoprotein family: general properties, biochemical and functional interaction with tubulin. Cell Struct Funct 1999, 24:345-357.

10. Curmi PA, Nogues C, Lachkar S, Carelle N, Gonthier MP, Sobel A, Lidereau R, Bieche I: Overexpression of stathmin in breast carcinomas points out to highly proliferative tumours. Br J Cancer 2000, 82:142-150.

11. Friedrich B, Gronberg H, Landstrom M, Gullberg M, Bergh A: Differentiationstage specific expression of oncoprotein 18 in human and rat prostatic adenocarcinoma. Prostate 1995, 27:102-109.

12. Gan L, Guo K, Li Y, Kang X, Sun L, Shu H, Liu Y: Up-regulated expression of stathmin may be associated with hepatocarcinogenesis. Oncol Rep 2010, 23:1037-1043

13. Jeon TY, Han ME, Lee YW, Lee YS, Kim GH, Song GA, Hur GY, Kim JY, Kim HJ, Yoon S, Baek SY, Kim BS, Kim BJ, Oh SO: Overexpression of stathmin1 in the diffuse type of gastric cancer and its roles in proliferation and migration of gastric cancer cells. Br J Cancer 2010, 102:710-718.

14. Singer $S$, Ehemann $V$, Brauckhoff $A$, Keith $M$, Vreden $S$, Schirmacher $P$, Breuhahn K: Protumorigenic overexpression of stathmin/Op18 by gain-of-function mutation in p53 in human hepatocarcinogenesis. Hepatology 2007, 46:759-768

15. Yuan RH, Jeng YM, Chen HL, Lai PL, Pan HW, Hsieh FJ, Lin CY, Lee PH, Hsu $\mathrm{HC}$ : Stathmin overexpression cooperates with p53 mutation and osteopontin overexpression, and is associated with tumour progression, early recurrence, and poor prognosis in hepatocellular carcinoma. J Pathol 2006, 209:549-558.

16. Lamberti A, Caraglia M, Longo O, Marra M, Abbruzzese A, Arcari P: The translation elongation factor $1 \mathrm{~A}$ in tumorigenesis, signal transduction and apoptosis: review article. Amino Acids 2004, 26:443-448.

17. Edmonds BT, Wyckoff J, Yeung YG, Wang Y, Stanley ER, Jones J, Segall J, Condeelis J: Elongation factor-1 alpha is an overexpressed actin binding protein in metastatic rat mammary adenocarcinoma. J Cell Sci 1996, 109(Pt 11):2705-2714.

18. Pecorari L, Marin O, Silvestri C, Candini O, Rossi E, Guerzoni C, Cattelani S, Mariani SA, Corradini F, Ferrari-Amorotti G, Cortesi L, Bussolari R, Raschella G, 
Federico MR, Calabretta B: Elongation Factor 1 alpha interacts with phospho-Akt in breast cancer cells and regulates their proliferation survival and motility. Mol Cancer 2009, 8:58.

19. Grassi G, Scaggiante B, Farra R, Dapas B, Agostini F, Baiz D, Rosso N, Tiribelli $C$ : The expression levels of the translational factors eEF1A $1 / 2$ correlate with cell growth but not apoptosis in hepatocellular carcinoma cell lines with different differentiation grade. Biochimie 2007, 89:1544-1552.

20. Edmondson HA, Steiner PE: Primary carcinoma of the liver: a study of 100 cases among 48,900 necropsies. Cancer 1954, 7:462-503.

21. Holmfeldt P, Sellin ME, Gullberg M: Upregulated Op18/stathmin activity causes chromosomal instability through a mechanism that evades the spindle assembly checkpoint. Exp Cell Res 2010, 316:2017-2026.

22. Zhang Y, Fujita N, Tsuruo T: Caspase-mediated cleavage of p21Waf1/Cip1 converts cancer cells from growth arrest to undergoing apoptosis. Oncogene 1999, 18:1131-1138.

doi:10.1186/1479-5876-12-154

Cite this article as: Ahn et al:: Increased expression of stathmin and elongation factor $1 a$ in precancerous nodules with telomere dysfunction in hepatitis B viral cirrhotic patients. Journal of Translational Medicine 2014 12:154.

\section{Submit your next manuscript to BioMed Central and take full advantage of:}

- Convenient online submission

- Thorough peer review

- No space constraints or color figure charges

- Immediate publication on acceptance

- Inclusion in PubMed, CAS, Scopus and Google Scholar

- Research which is freely available for redistribution 